\title{
Molecular Dynamics Investigations of Lipid Langmuir Monolayers Using a Coarse-Grain Model
}

\author{
Steve O. Nielsen, ${ }^{\dagger}$ Carlos F. Lopez, ${ }^{\dagger}$ Preston B. Moore, ${ }^{\dagger}$ John C. Shelley, ${ }^{\S}$ and \\ Michael L. Klein*,† \\ Center for Molecular Modeling and Department of Chemistry, University of Pennsylvania, Philadelphia, \\ Pennsylvania 19104, Department of Chemistry and Biochemistry, University of the Sciences in Philadelphia, \\ 600 South 43rd Street Philadelphia, Pennsylvania 19104, and Schrödinger Inc., 1500 South West First Avenue, \\ Suite 1180, Portland, Oregon 97201
}

Received: May 8, 2003; In Final Form: September 2, 2003

\begin{abstract}
We present the results of molecular dynamics (MD) simulations of coarse-grain 1,2-di- $n$-alkanoyl-sn-glycero3-phosphocholine Langmuir monolayer systems. Two separate alkanoyl length lipids are considered. Surface pressure versus area per lipid isotherms are obtained for both lipid species and are compared to experimental and previous atomistic MD work. The short-tail lipid cannot support a surface pressure above that expected at the critical micelle concentration. In contrast, the long-tail lipid is studied in the metastable regime of slightly negative surface tension. At still higher surface coverage, the interface is destabilized, leading to monolayer collapse via a novel mechanism in which a charge-neutralizing bridge of zwitterionic headgroups is established to transport lipids across the alkanoyl region. Area per lipid compression simulations are also performed at constant lipid number to demonstrate that the observed instabilities are generic.
\end{abstract}

\section{Introduction}

A Langmuir monolayer (LM) is formed when amphiphilic molecules orient at an air-water interface. Dynamic and structural properties of LMs have been studied using a variety of techniques including fluorescence, Brewster angle microscopy, infrared-Raman spectroscopy, X-ray and neutron scattering, and nonlinear optical approaches. ${ }^{1}$ A wide spectrum of dynamical events occur at different time and length scales in monolayers, from ordering and phase transitions in two dimensions through surface undulations and capillary waves to collapse transitions in three dimensions. Theoretical work on the structure and dynamics of LMs has been mostly aimed at pure surfactant-air-water systems and their relation to experiment. $^{2-8}$

LMs are usually studied as a function of surface coverage. Experimentally, one monitors the surface pressure, $\pi=\gamma_{0}-$ $\gamma$, where $\gamma_{0}$ is the surface tension of the pure air-water system and $\gamma$ is the resultant surface tension in the presence of surfactant. Pressure-area isotherms can be mapped out and frequently show hysteresis loop behavior as some of the surfactant is lost because of irreversible monolayer collapse. ${ }^{9}$

At high coverage, the system tends to increase its interfacial area by moving away from a flat geometry, eventually leading to collapse of the monolayer. ${ }^{10}$ In fact, the amplitude of the thermal fluctuations diverges as the surface tension approaches $0\left(\pi\right.$ approaches $\left.\gamma_{0}\right) .{ }^{11}$ Milner $^{12}$ showed that an ideal diblock copolymer monolayer develops a buckling instability only at zero surface tension. Collapse usually occurs before this limit. However, in the work by Schief ${ }^{11}$ on dipalmitoyl phosphatid-

* To whom correspondence should be addressed. E-mail: klein@ cmm.upenn.edu. URL: www.cmm.upenn.edu.

University of Pennsylvania.

$\doteqdot$ University of the Sciences in Philadelphia.

$\S$ Schrödinger Inc. ylcholine (DPPC) monolayers, collapse is not observed until the pressure exceeds $71 \mathrm{dyn} / \mathrm{cm}$ (with $\gamma_{0}=72$ ). Collapse can occur into the solvent subphase or outward on top of the monolayer. The outward collapse is by the formation of multilayers. ${ }^{13}$ These multilayers can be oriented in various ways; one possibility is the formation of a trilayer by a "rollover" mechanism in which a bilayer is formed on top of the monolayer. ${ }^{14}$ If the bulk solution in equilibrium with the monolayer is at or above its critical micelle concentration (CMC), the monolayer may shed micelles readily into the bulk. ${ }^{12}$ An extensive analysis of contributions to the bending free energy has been undertaken by $\mathrm{Hu}^{2}$

In the present paper, we explore the validity of our model for LM systems by examining pure lipid-air-water systems for two situations motivated by these two examples. The effect of both short- and long-tail phospholipid packing at the air/ water interface is examined with equilibrium simulations performed at fixed area per lipid. Density profiles normal to the interface are discussed in section IIIA, and order parameters are computed in section IIIB. The surface pressure versus area per lipid isotherms are mapped out in section IIIC and compared to experimental and simulation data. In section IIID, the study is extended into the region of dynamical instability, which develops when the packing is too dense. The instability that occurs at high surface coverage is probed in two different ways. First, simulations are done at fixed cross-sectional unit cell area with differing numbers of lipids. Second, compression simulations are done in which the number of lipids is fixed while the cross-sectional area of the box is decreased isothermally starting from a low surface coverage.

The two mechanisms that we observe whereby the monolayer relieves its high surface pressure are either through the development of curvature to increase the interfacial area or through a loss of headgroups to the exterior of the monolayer via a lipidic bridge transport mechanism. This bridge minimizes the energy 


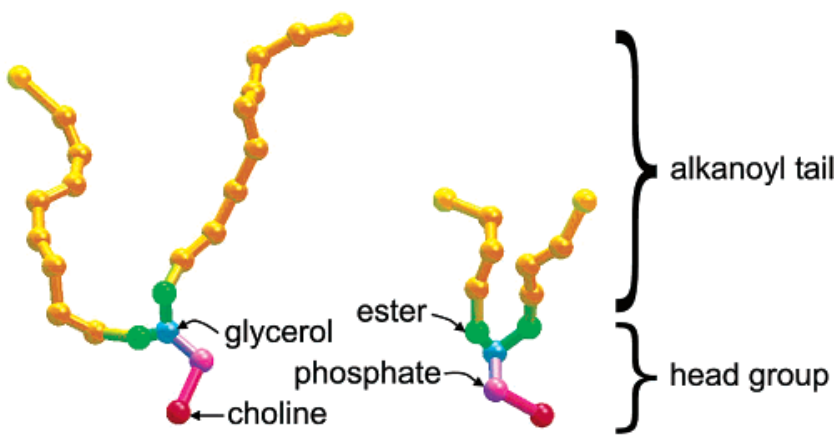

Figure 1. Thirteen site model of 1,2-di-tetradecanoyl-sn-glycero-3phosphocholine $\left(\mathrm{DC}_{14} \mathrm{PC}\right.$, commonly referred to as dimyristoylphosphatidylcholine) and 23 site model of $\mathrm{DC}_{29} \mathrm{PC}$. The choline and phosphate sites carry positive and negative electrostatic charges, respectively.

penalty for the headgroups traversing the hydrophobic region by transporting them along a charge-neutralizing pathway constructed from zwitterionic headgroups. After enough lipids are transported to the exterior, the monolayer stabilizes and flattens.

\section{Method}

A 13 site model of 1,2-di-tetradecanoyl-sn-glycero-3-phosphocholine has been developed and parametrized for a bilayer environment. ${ }^{16-21}$ This molecule is commonly known as dimyristoylphosphatidylcholine (DMPC), but for generality, we refer to it as $\mathrm{DC}_{14} \mathrm{PC}$, emphasizing the tetradecane-based tail. Briefly, one interaction site represents the positively charged choline group, and another site represents the negatively charged phosphate group. The alkanoyl tails and remaining atoms are modeled by sites representing parts of the lipid of similar size as shown in Figure 1. In analyzing the data, the headgroup is defined to be the center of mass of the choline, phosphate, and glycerol sites. Three and four of the alkane tail units are used to construct nonane and dodecane, respectively. The long-tail lipid, $\mathrm{DC}_{29} \mathrm{PC}$, was generated from the existing lipid by the addition of five tail units to each of the two alkanoyl chains (see Figure 1). The CG water site represents a loose grouping of three water molecules, but potentials of mean force are also used to capture some of the atomistic solvent shell structure. This CG model has been shown to self-assemble into bilayer, inverse hexagonal, and Langmuir monolayer phases and has been extended to work in the NPT ensemble. ${ }^{21}$ In addition to having fewer sites than the corresponding all-atom representation, the soft interaction potentials allow for a larger time step and result in enhanced diffusional and rotational motion for the lipid species. ${ }^{19,20}$ The simulation times reported in this paper are the raw values given by the product of the time step and the number of steps taken. Diffusional motion for the lipid species occurs 2 orders of magnitude faster than that in the allatom (AA) case. This is because of two factors. First, the soft intermolecular potentials, which automatically result from coarse graining, allow particles to slide by each other easily. Second, because the hydrogen-bonding network at the lipid/water interface is captured in a mean-field manner, the lateral motion of lipids in the membrane plane is not inhibited by hydrogenbonding dynamics. A raw simulation time of 500 ps corresponds roughly to $50 \mathrm{~ns}$ of an AA simulation. ${ }^{17,19,20}$

The geometry employed in the molecular dynamics studies consists of a slab of liquid, infinite through periodic boundary conditions in the $x y$ plane, with vacuum at either end. This construction results in two air-water interfaces; enough water is used so that there is a bulk water environment in the middle of the simulation cell separating the two interfaces (see Figure $3)$. Three-dimensional periodic boundary conditions facilitate the computation of Ewald sums, ${ }^{22}$ but the $z$-direction is chosen to be large so that the slabs do not interact with their periodic images.

An equilibrated slab of pure water was generated and a single lipid molecule configuration was prepared for each of the shortand long-lipid species. Then, for a run with $N$ lipids in a box of cross-sectional size $L \times L$, the lipids were placed on an $x y$ grid of spacing $L\left\lceil(N / 2)^{1 / 2}\right\rceil$ in each direction, where $\lceil a\rceil$ rounds $a$ up to the nearest integer. This leaves $\left\lceil(N / 2)^{1 / 2}\right\rceil-N / 2$ grid positions unfilled. Half of the lipids are placed at these $x y$ positions and at a $z$-position and orientation such that the headgroups are in water and the tails are in a vacuum at one of the two interfaces. The other half of the lipids are placed at these same $x y$ positions but at a $z$-position and orientation such that the headgroups are in water and the tails are in a vacuum at the other interface. The lipids are held in place, while the water is allowed to relax around them. The entire system is allowed to equilibrate before the production run.

All simulations except the compression runs used a box length of $L=70 \AA$. Single-component simulations of water $(2725$ molecules), nonane (1024 molecules), and dodecane (1024 molecules) were run for 500 ps with a 50 ps equilibration period. Short-lipid ( $\mathrm{DC}_{14} \mathrm{PC}$ ) runs were performed with 2725 waters and, separately, $N=10,20,40,60$, and 80 lipids for $500 \mathrm{ps}$ with a 50 ps equilibration period and with 2725 waters and $N$ $=100,120,130,140$, and 160 lipids for 500 ps with a 550 ps equilibration period. Long-lipid $\left(\mathrm{DC}_{29} \mathrm{PC}\right)$ runs were performed with 2725 waters and, separately, $N=10,20,40,60,80,100$, $120,130,140,150$, and 160 lipids for 500 ps with a 50 ps equilibration period and with 5000 waters and $N=180,200$, $220,240,260$, and 280 lipids for 500 ps with a 50 ps equilibration period. Finally, compression simulations with 20000 waters and $N=500$ long lipids were run for $350 \mathrm{ps}$ with a 30 ps equilibration period starting with a box of $L=$ $190 \AA$ and with, separately, applied pressures of $0.5,1.5$, and $4.5 \mathrm{~atm}$. In total, 33 simulations were performed. The computer time required for this study is as follows. For the system consisting of 140 long lipids and 2725 water particles, a total of 5945 particles, $7440 \mathrm{~s}$ are required to simulate a $100 \mathrm{ps}$ trajectory on a single processor $2.8 \mathrm{GHz}$ Pentium4. The entire study consumed $475 \mathrm{~h}$ of computer time.

All systems are run in the NVT ensemble $(303.15 \mathrm{~K})$ except the $N=500$ systems, which are run in the NPT ensemble $(P=$ $0.5,1.5$, and $4.5 \mathrm{~atm}, T=303.15 \mathrm{~K}$ ) with an isotropic barostat to preserve the square cross-sectional box geometry. The temperature (pressure) is controlled using Nosé-Hoover thermostat (barostat) chains of length four. ${ }^{23}$ The van der Waals cutoff was set at $19 \AA$. The long-range electrostatic interactions are computed with Ewald sums. The instantaneous surface tension is computed as

$$
\gamma_{\text {inst }}=\frac{L_{z}}{2}\left(P_{z z}-\frac{P_{x x}+P_{y y}}{2}\right)
$$

where the first factor of $1 / 2$ is included to account for the two interfaces in the simulation box, $L_{z}$ is the box size in the $z$-direction, and $P_{i j}$ is the $i j$ component of the pressure tensor. The monolayer plane is the $x y$ plane.

\section{Results and Discussion}

A. Equilibrium Density Profiles Normal to Interface. The equilibrium lipid density profiles are shown in Figure 2 for a range of surface coverages. The short-lipid systems are shown 

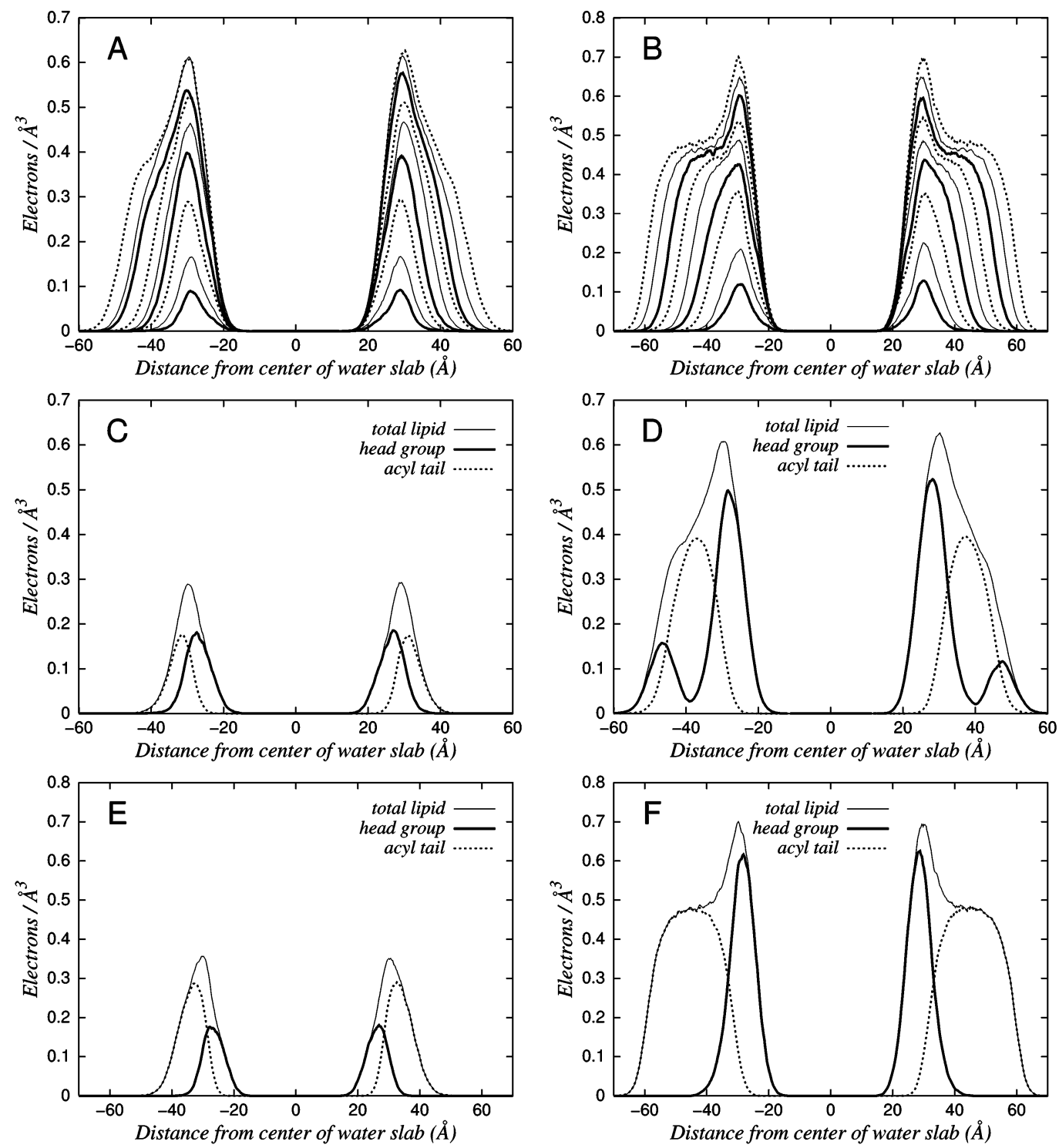

Figure 2. Equilibrium density profile of the lipids normal to the monolayer interface. Panel A displays the short-lipid density, while panel B depicts the long lipid. There are nine curves in each of these panels, which correspond, in order of increasing height, to the number of lipids at each interface being 5 (thick line), 10 (thin line), 20 (dotted line), 30 (thick line), 40 (thin line), 50 (dotted line), 60 (thick line), 70 (thin line), and 80 (dotted line). The 20 and 80 lipid per interface plots of panels A and B are shown in more detail in panels C and D for the short lipid and panels $\mathrm{E}$ and $\mathrm{F}$ for the long lipid, respectively. The total lipid density is repeated in thin line. In dotted line is the tail density (including the ester groups), and in thick line is the headgroup density. Notice that the headgroups in panel D have a bimodal distribution, indicating that there are lipids occupying the space exterior to the monolayer leaflet, forming a partial bilayer.

in Figure 2A and the long-lipid systems in Figure 2B. The maximum remains in the same location across all coverages and is associated with the headgroups. The shoulder that develops at high coverage is largely due to the alkanoyl tails, which align perpendicular to the monolayer plane. This is illustrated for the long lipid in Figures 2E,F, and 3. But these density profiles provide a misleading picture for the short lipid as seen in Figure 2C,D, where the lipid electron density is decomposed into contributions from the headgroup and alkanoyl tail regions. It is seen in Figure 2D that some of the apparent tail ordering is actually the result of a population of lipids positioned to the exterior of the monolayer with their tails facing those in the monolayer. This inverted population occurs when short lipids rotate out of the monolayer, keeping their tails intertwined with the monolayer tails. In the present CG model, the interaction between the lipid headgroup and water sites is relatively weak. ${ }^{20}$ This is at least partially due to the lack of hydrogen bonding at the interface. Consequently, the cost of rotating the lipid and maintaining a hydrophobic interaction with the monolayer while relieving the pressure at the interface is not greatly unfavorable compared to remaining in the monolayer. The translational and rotational motion of the CG lipids exacerbate this effect due to the enhanced sampling of configuration space. ${ }^{20} \mathrm{We}$ feel this behavior is an artifact of the model. For the long-tail lipids, the barrier for such flipping events is larger because the headgroups must traverse a wider hydrocarbon region. Anticipating some of our results, we find that a cooperative mechanism among many lipids must exist for the long-tail species to move to the exterior region; for the positive and slightly negative surface tensions studied, the interface is stable (see Figure 2E,F).

B. Order Parameters. The extent of ordering in the alkanoyl chains can be characterized by an order parameter given by the correlation of unit vectors $\mathbf{s}_{i}$ defined from the headgroup to the terminal carbon site of each of the two tails, ${ }^{25}$

$$
\mathbf{s}_{\text {relative }}=\left\langle\sum \mathbf{s}_{i} \cdot \mathbf{s}_{j}\right\rangle
$$



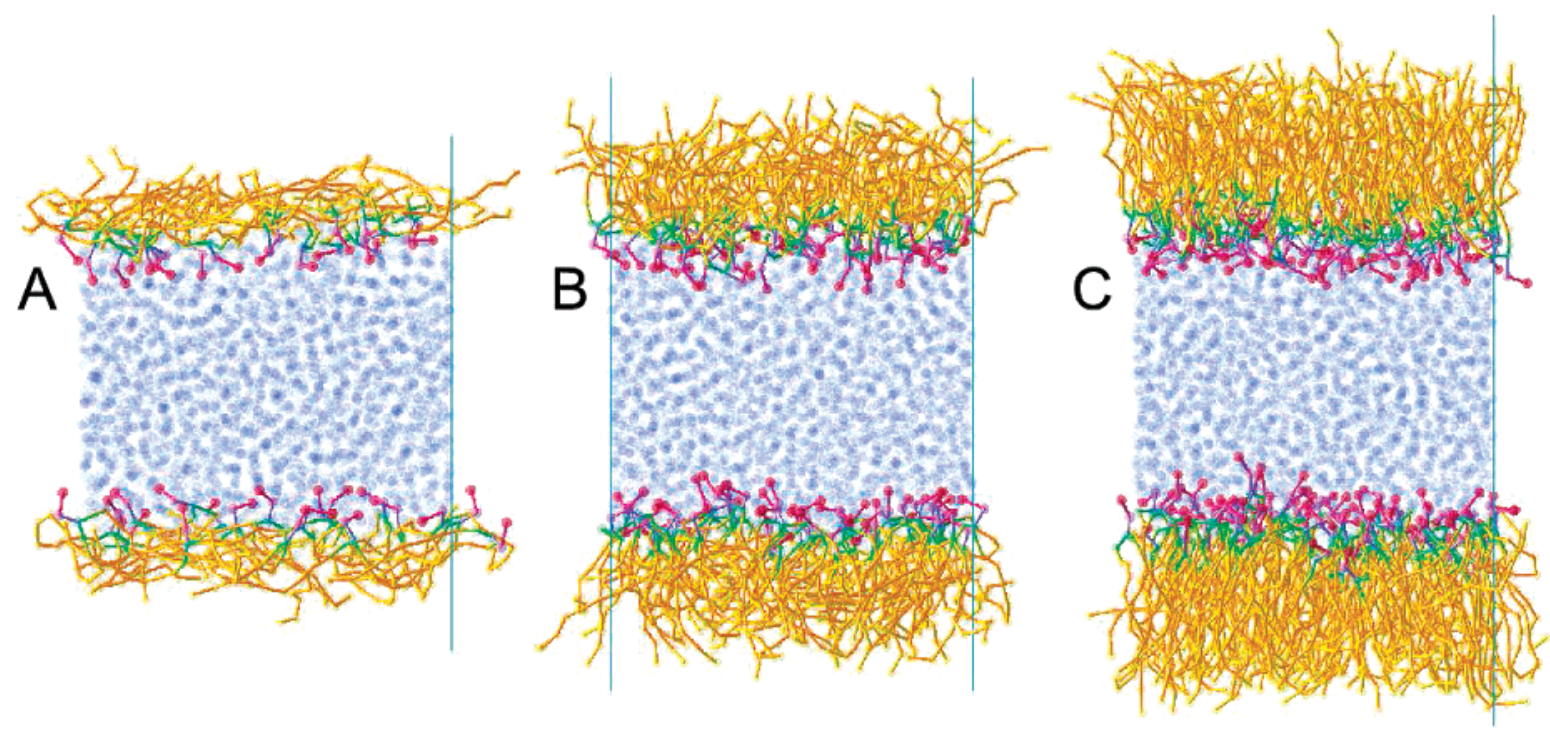

Figure 3. Snapshots of the long-lipid system at 245 (A), 98 (B), and 61 (C) $\AA^{2}$ area per lipid at equilibrium, corresponding to 20, 50 , and 80 lipids per interface, respectively. The alkanoyl tails order and stand up at high coverage. Coloring is as follows: water blue; lipid as in Figure 1.

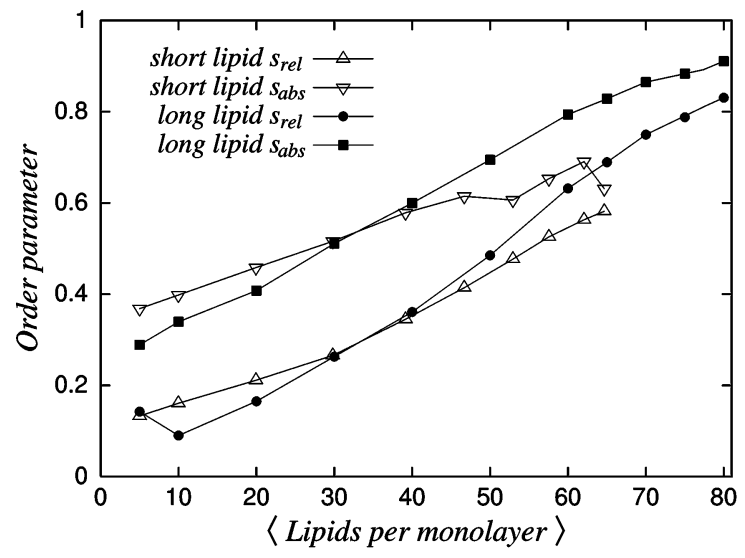

Figure 4. The relative and absolute order parameters of eqs 2 and 3 are plotted versus the average number of lipids per monolayer for the short- and long-tail lipid species. The inverted short-lipid population is not included. The data points are connected with straight line segments solely as a guide for the eye.

where the prime on the summation denotes that we exclude correlations between vectors associated with the same molecule and between the two monolayers; in addition, we exclude lipids that occupy the region exterior to the monolayer leaflet. By excluding this inverted population, we only count the number of lipids in the monolayers themselves. This is reflected in the values shown on the ordinate of Figure 4. Note that the headtail correlation function would be decreased in magnitude by having an outer leaflet because of a sign cancellation between molecules in the same leaflet and in different leaflets (see section IIIA). For the long lipid, the lowest-coverage system studied is anomalous. The order parameter is higher than the trend would suggest because the lipids cluster together to lower their potential energy, overriding the entropic benefit of spreading on the surface. The tails of the short lipid have a much lower maximal van der Waals interaction energy; for the short lipid, the entropic surface spreading dominates at very low coverage. An analogy can be made to liquid-vapor coexistence. The short lipids behave as if they are either supercritical or in the gas phase. The long lipids behave as if they are subcritical and in the coexistence region with the gas density close to zero. This suggests that these models could perhaps be used to study rafting phenomena.
An absolute rather than relative order parameter is also calculated. Here the angle with respect to the normal vector to the appropriate monolayer plane $\hat{n}$ is measured for each of the head to tail vectors defined above,

$$
\mathbf{s}_{\text {absolute }}=\left\langle\sum \mathbf{s}_{i} \cdot \hat{n}\right\rangle
$$

where the prime on the summation denotes that we exclude lipids that occupy the region exterior to the monolayer leaflet. A lipid is excluded if its headgroup center of mass is more than a few standard deviations away from the headgroup density maximum (see Figure 2). However, the inverted population is in dynamic equilibrium with the monolayer, so this criterion is time-dependent. The high-coverage short-lipid $\mathbf{s}_{\text {abs }}$ values are affected by these interchanging lipids because they are rotating so are not oriented perpendicular to the monolayer plane.

The order parameters shown in Figure 4 demonstrate that in Langmuir monolayer systems there is an additional thermodynamic variable at our disposal: the surface pressure. The surface pressure is in one-to-one correspondence with the surface coverage (area occupied per lipid) as shown in Figure 5 (Table 1 provides data not shown on the scale of the plot of Figure 5). This additional control over the system properties is exploited by experimentalists to better understand lipid-protein interactions. ${ }^{15}$

C. Surface Pressure versus Area per Lipid Isotherms. To calculate surface pressure, we must know the air/water surface tension $\left(\gamma_{0}\right)$ for our model. We will also make use of the air/ hydrocarbon surface tension in what follows. The CG water and hydrocarbon nonbonded parameters were determined on the basis of experimental bulk density and vapor pressure data. ${ }^{16}$ The pure species are prepared in a slab with vacuum at either end as described in section II. The surface tension is computed by averaging $\gamma_{\text {inst }}$ [eq 1] over the length of the simulation. The CG model gives a surface tension at the air/water interface of $72 \pm 1 \mathrm{dyn} / \mathrm{cm}$ at $303.15 \mathrm{~K}$. This value agrees with the experimental value of $71.2 \mathrm{dyn} / \mathrm{cm}$. For hydrocarbons at 303.15 $\mathrm{K}$, we obtain a surface tension of $\gamma=18.0 \pm 1.5 \mathrm{dyn} / \mathrm{cm}$ for nonane and $\gamma=17.5 \pm 2.0 \mathrm{dyn} / \mathrm{cm}$ for dodecane. The experimental value ${ }^{24}$ for the surface tension of nonane is 22.0 $\mathrm{dyn} / \mathrm{cm}$; our model is reasonable but could use improvement in this respect. 

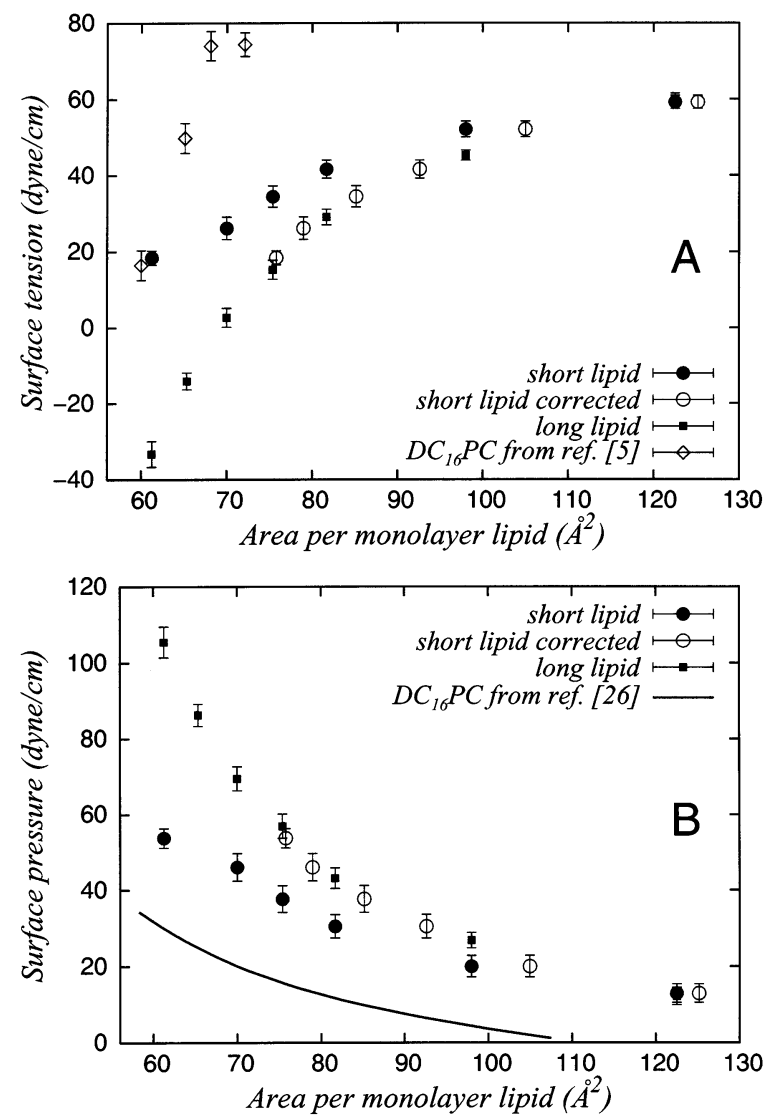

Figure 5. Surface tension (A) and surface pressure (B) versus area per lipid. In panel A, the short- and long-tail lipid systems of the present study are compared to a constant surface tension ensemble all-atom MD result of Feller ${ }^{5}$ for $\mathrm{DC}_{16} \mathrm{PC}$ at $323 \mathrm{~K}$. In panel $\mathrm{B}$, the short- and long-tail lipid systems of the present study are compared with the experimental result of Somerharju ${ }^{26}$ for $\mathrm{DC}_{16} \mathrm{PC}$ at $321 \mathrm{~K}$. The inverted short-lipid population is not included in the value on the ordinate for the "corrected" data, but the surface tension/pressure is that of the entire system. Error bars are assigned from the standard deviation of the mean in each of 10 consecutive 50 ps windows. The error bars for the Feller data are as reported. Data not shown on the scale of the plot can be found in Table 1.

The surface tension and pressure versus area per lipid isotherms are shown for both lipid species in Figure 5. The inverted population of short lipids does not contribute to the ordinate values for the "corrected" data shown in Figure 5, but the surface tension/pressure is that of the entire system. The
TABLE 1: Data off the Scale of Figure 5

\begin{tabular}{|c|c|c|c|}
\hline $\begin{array}{l}\text { lipids per } \\
\text { interface }\end{array}$ & $\begin{array}{c}\text { area per } \\
\text { lipid }\left(\AA^{2}\right)\end{array}$ & $\begin{array}{c}\text { surface } \\
\text { tension } \\
(\text { dyn/cm) }\end{array}$ & $\begin{array}{c}\text { surface } \\
\text { pressure } \\
(\mathrm{dyn} / \mathrm{cm})\end{array}$ \\
\hline \multicolumn{4}{|c|}{ Short Lipid $\mathrm{DC}_{14} \mathrm{PC}$} \\
\hline 30 & 163 & $66.7 \pm 1.2$ & $5.5 \pm 1.9$ \\
\hline 20 & 245 & $69.8 \pm 1.5$ & $2.4 \pm 2.2$ \\
\hline 10 & 490 & $72.0 \pm 1.3$ & $0.1 \pm 2.0$ \\
\hline 5 & 980 & $72.3 \pm 1.4$ & $-0.2 \pm 2.1$ \\
\hline \multicolumn{4}{|c|}{ Long Lipid $\mathrm{DC}_{29} \mathrm{PC}$} \\
\hline 30 & 163 & $67.4 \pm 1.2$ & $4.7 \pm 1.9$ \\
\hline 20 & 245 & $70.2 \pm 2.5$ & $2.0 \pm 3.2$ \\
\hline 10 & 490 & $71.4 \pm 1.0$ & $0.7 \pm 1.7$ \\
\hline 5 & 980 & $72.6 \pm 1.2$ & $-0.5 \pm 1.9$ \\
\hline
\end{tabular}

true short-lipid isotherm lies somewhere between the corrected and uncorrected data sets. The pressure-area isotherm for the short-lipid species hence lies below that for the long-lipid species, as it must.

A previous all-atom MD simulation reported the surface tension versus area per lipid in a $\mathrm{DC}_{16} \mathrm{PC}$ monolayer ${ }^{5}$ and gave the correct trend of increasing surface tension with increasing area, but the resulting curve is too stiff compared with the experimental result ${ }^{26}$ at $321.15 \mathrm{~K}$ (see Figure 5). More recent fully atomistic MD results ${ }^{6}$ are not any closer to reproducing experimental data. The area per lipid is $15 \%-20 \%$ too large in the CG model, which contributes to the offset compared to the experimental curve (see Figure 5).

In the case of the short lipid, it is observed that the monolayer cannot support a coverage much below $75 \AA^{2}$ per lipid. Putting more lipids into the monolayer destabilizes the interface and causes lipids to populate the region exterior to the leaflet.

The surface tension of surfactants at the critical micelle concentration (CMC) is often close to that for a bulk hydrocarbon liquid/vapor interface. To relate this limiting value to the CMC, we computed the surface tension of both nonane and dodecane (see above). The values of $\gamma=18.0 \pm 1.5 \mathrm{dyn} / \mathrm{cm}$ for nonane and $\gamma=17.5 \pm 2.0 \mathrm{dyn} / \mathrm{cm}$ for dodecane fall in a band just below the surface tension of the highest-coverage point in Figure 5 of $18.4 \pm 1.9 \mathrm{dyn} / \mathrm{cm}$. This leads us to conclude that the short lipid cannot sustain a monolayer coverage greater than that which corresponds to the surface tension at the CMC.

The long lipid reaches zero surface tension at an area of about $69 \AA^{2}$ per lipid. This is close to the equilibrium lamellar $L_{\alpha}$ phase area per lipid of $75 \AA^{2} .{ }^{27}$ The smallest area per lipid systems display negative surface tension, indicating a metastable state.
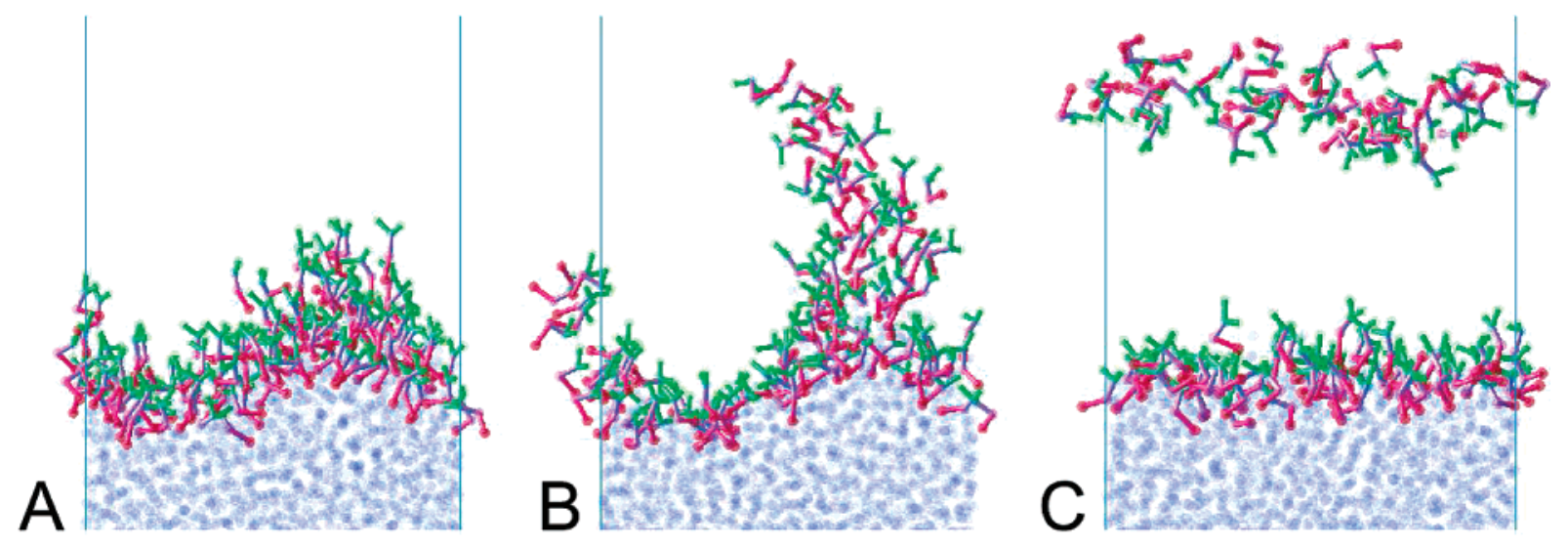

Figure 6. Monolayer instability and collapse shown at one interface of the 120 long lipid per interface system. The initially flat interface (not shown) develops curvature (A) and then opens a bridge to the exterior of the leaflet (B); the bridge transports enough material to eventually bring the system back into equilibrium with a flat monolayer interface (C). See Figure 7 for the time and the surface tension of these snapshots. Alkanoyl tails are not shown. Coloring is as follows: water blue; lipid as in Figure 1. 


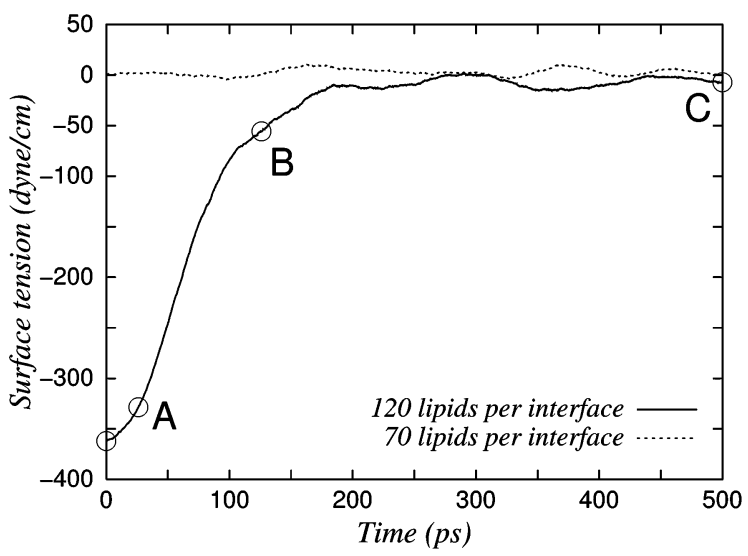

Figure 7. Instantaneous surface tension versus raw simulation time for the 120 long lipid per interface unstable system shown in Figure 6. The data are smoothed with a $100 \mathrm{ps}$ wide symmetric second-order Savitzky-Golay filter. ${ }^{28}$ Shown for comparison is the corresponding 70 long lipid per interface curve, which has a surface tension of roughly zero. The four points marked on the plot are as follows from left to right. The first point corresponds to the initially flat 120 lipid per interface monolayer. The second point corresponds to Figure 6A when the interface has developed curvature. The third point corresponds to Figure 6B when the monolayer is expelling lipids. The fourth point corresponds to Figure $6 \mathrm{C}$ when the monolayer has come to equilibrium. The reader is reminded that the effective time is roughly 2 orders of magnitude larger than the raw simulation time.

D. Unstable Systems. We performed simulations with the long alkanoyl lipid species at higher surface coverage to explore the negative surface tension metastability and to push it into an unstable regime. Additional water sites are used to ensure that the interfaces are well separated even when they develop curvature (see section II).

The periodic boundary conditions employed limit the development of curvature in the monolayer. This geometrical constraint restricts the interfacial area increase that comes with developing a nonplanar geometry and artificially stabilizes the systems of section IIIC down to moderate negative surface tension values. At higher coverage, the two mechanisms that we observe whereby the monolayer relieves its high surface pressure are either through the development of curvature (see
Figures $6 \mathrm{~A}$ and $8 \mathrm{~B}$ ) to increase the interfacial area or through a loss of headgroups to the exterior of the monolayer by a bridge transport mechanism, as seen in Figures 6A and 8B. The bridge enables the zwitterionic lipid headgroups to maintain electrostatic contacts with one another as they traverse the hydrophobic region. The bridge hence acts as a charge-neutralizing pathway. After enough lipids are transported to the exterior, the monolayer heals back to a flat geometry (see Figure 6C). For the 120 lipid run shown in Figure 6, the instantaneous surface tension versus time is shown in Figure 7. This plot clearly shows the system relaxing to equilibrium. There is also a suggestion of a different relaxation regime during the bridge transport process, as evidenced by the shoulder at point B in Figure 7.

To mimic experiments in which the surface pressure is controlled and to validate our results with a different simulation method, we took an initially diffuse system and decreased the area per lipid at three different rates by shrinking the crosssectional box size through an externally applied pressure. The cross-sectional box geometry was constrained to be square throughout this compression. The system is driven into the negative surface tension regime shortly after $100 \mathrm{ps}$ in all three cases. The monolayers develop instabilities similar to those seen in the constant area runs, but the greater number of lipids and larger box size allows the bending instability to occur earlier and the extent of bending to develop to much greater curvatures (see Figure 8B) than is seen with the small box size of Figures 6 and 8A. Even this large system is artificially stabilized by the period boundary conditions. The extreme curvature of the interface in Figure 8B is suggestive of the budding of lipid aggregates into the bulk water subphase. At much larger system sizes, we would expect to observe part of the LM collapsing into the solvent.

\section{Conclusions}

Equilibrium studies of Langmuir monolayers of $\mathrm{DC}_{14} \mathrm{PC}$ and $\mathrm{DC}_{29} \mathrm{PC}$ were performed at different surface coverages. The lipid tails were seen to order and stand up with increasing coverage. The full surface pressure versus area per lipid isotherms were constructed and compared to experimental results. The longtail species is of interest when studying a transmembrane peptide
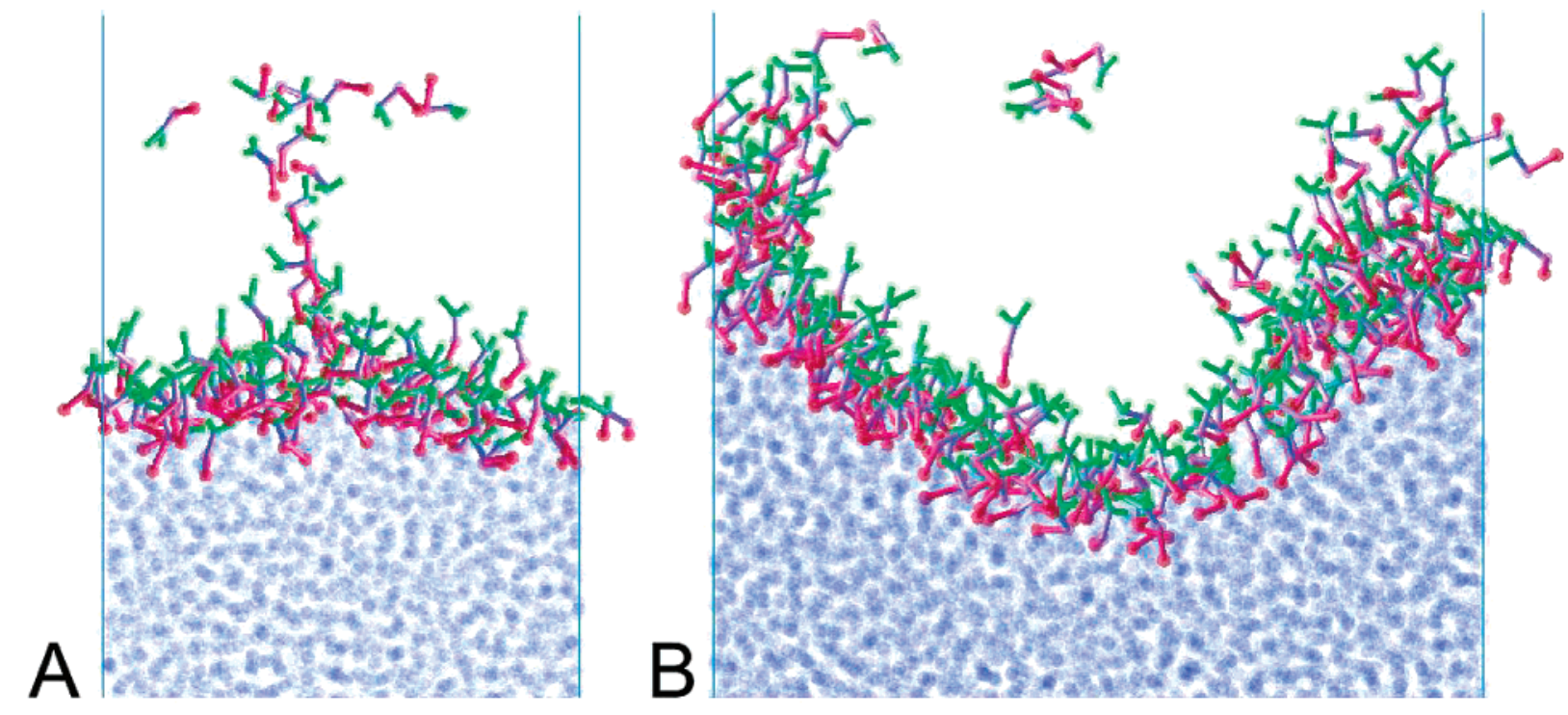

Figure 8. Panel A shows the 100 long lipid per interface system at $400 \mathrm{ps}$. A headgroup bridge has been established to transport lipids to the region exterior to the monolayer. The system is only moderately unstable, and the monolayer is almost flat. Panel B shows the 250 long lipid per interface compression run of $4.5 \mathrm{~atm}$ at $350 \mathrm{ps}$. The larger system size allows for a bigger curvature to develop. Only one of the two interfaces is shown in each case. Alkanoyl tails are not shown. Coloring is as follows: water blue; lipid as in Figure 1. 
embedded in a Langmuir monolayer because the wide singletail hydrocarbon region is similar in width to the hydrophobic region of a standard bilayer; we have begun these studies. The dynamic instability that occurs at high coverage was explored, and monolayer collapse to the exterior of the interface was observed to occur via a bridge transport mechanism in which headgroups form a charge-neutralizing bridge across the alkanoyl region. After lipids have been transported across the hydrophobic region, the bridge structure breaks apart, and the final system consists of a stable lipid monolayer at a surface tension of roughly zero and an exterior lipid layer the tails of which face the monolayer tails.

Computer simulations of Langmuir monolayers must balance the level of detail describing the hydrogen-bonding and phospholipid headgroup electrostatic network at the air/water interface with the desired time and spatial scales. Fully atomistic classical molecular dynamics (MD) results are far from reproducing experimental surface pressure versus area per lipid isotherms. 5,6 The coarse-grain (CG) method employed in the present work uses a parametrization strategy encompassing both all-atom simulations and experimental observables ${ }^{16}$ and does a much better job of reproducing the experimental surface tension of water, alkane, and phospholipid/water systems at the air/water interface. The decrease in computational cost by 5 orders of magnitude ${ }^{19,20}$ over all-atom MD makes the CG method feasible for mapping out the full surface pressure versus area per lipid isotherms and for studying instabilities that occur at high surface coverage. The limitations of the current $\mathrm{CG}$ model in describing Langmuir monolayers point the way to improving the model. In particular, the long-range variation of the headgroup-headgroup potentials is not likely correct, and the headgroup-water interactions are probably too weak. This is a normal part of the process for model development and refinement. This work is not only an experiment to learn something about the systems under study but also one to exercise the model itself and learn something about its capacity. The observed instabilities should be tested by doing limited fully atomistic simulations.

Acknowledgment. This work was supported in part by a grant from the Natural Sciences and Engineering Research Council of Canada and the National Institutes of Health. Discussion with Bin Chen and Goundla Srinivas is gratefully acknowledged.

\section{References and Notes}

(1) Dynarowicz-Lątka, P.; Dhanabalan, A.; Oliveira, O. N., Jr. Adv. Colloid Interface Sci. 2001, 91, 221-293.

(2) Hu, J.-G.; Granek, R. J. Phys. II (France) 1996, 6, 999-1022.

(3) Schmid, F.; Stadler, C.; Lange, H. Colloids Surf., A 1999, 149, 301-306.

(4) Marsh, D. Biochim. Biophys. Acta 1996, 1286, 183-223.

(5) Feller, S. E.; Zhang, Y.; Pastor, R. W. J. Chem. Phys. 1995, 103, 10267-10276.

(6) Kaznessis, Y. N.; Kim, S.; Larson, R. G. Biophys. J. 2002, 82, $1731-1742$.

(7) Okamura, E.; Fukushima, N.; Hayashi, S. Langmuir 1999, 15, 3589-3594.

(8) Dhathathreyan, A.; Collins, S. J. Langmuir 2002, 18, 928-931.

(9) Lipp, M. M.; Lee, K. Y. C.; Takamoto, D. Y.; Zasadzinski, J. A.; Waring, A. J. Phys. Rev. Lett. 1998, 81, 1650-1653.

(10) Ridsdale, R. A.; Palaniyar, N.; Possmayer, F.; Harauz, G. J. Membr. Biol. 2001, 180, 21-32.

(11) Schief, W. R.; Touryan, L.; Hall, S. B.; Vogel, V. J. Phys. Chem. B 2000, 104, 7388-7393.

(12) Milner, S. T.; Joanny, J.-F.; Pincus, P. Europhys. Lett. 1989, 9, 495-500.

(13) Ybert, C.; Lu, W.; Möller, G.; Knobler, C. M. J. Phys. Chem. B 2002, 106, 2004-2008.

(14) Ybert, C.; Lu, W.; Möller, G.; Knobler, C. M. J. Phys.: Condens. Matter 2002, 14, 4753-4762.

(15) Zheng, S.; Strzalka, J.; Ma, C.; Opella, S. J.; Ocko, B. M.; Blasie, J. K. Biophys. J. 2001, 80, 1837-1850.

(16) Shelley, J. C.; Shelley, M. Y.; Reeder, R. C.; Bandyopadhyay, S.; Klein, M. L. J. Phys. Chem. B 2001, 105, 4464-4470.

(17) Shelley, J. C.; Shelley, M. Y.; Reeder, R. C.; Bandyopadhyay, S.; Moore, P. B.; Klein, M. L. J. Phys. Chem. B 2001, 105, 9785-9792.

(18) Shelley, J. C. Modified coarse grain parameters for MD simulations in the NPT ensemble. Available through the web at www.cmm.upenn.edu/ $\sim$ moore/code/code.html (accessed 11/11/03).

(19) Lopez, C. F.; Moore, P. B.; Shelley, J. C.; Shelley, M. Y.; Klein, M. L. Comput. Phys. Commun. 2002, 147, 1-6.

(20) Lopez, C. F.; Nielsen, S. O.; Moore, P. B.; Shelley, J. C.; Klein,

M. L. J. Phys.: Condens. Matter 2002, 14 9431-9444.

(21) Nielsen, S. O.; Klein, M. L. In Bridging the Time Scales: Molecular Simulations for the Next Decade; Nielaba, P., Mareschal, M., Ciccotti, G., Eds.; Springer: Berlin, 2002; pp 27-63.

(22) Hautman, J.; Klein, M. L. Mol. Phys. 1992, 75, 379-395.

(23) Martyna, G. J.; Klein, M. L.; Tuckerman, M. J. Chem. Phys. 1992 97, 2635-2643

(24) Rudek, M. M.; Fisk, J. A.; Chakarov, V. M.; Katz, J. L. J. Chem. Phys. 1996, 105, 4707-4713.

(25) Boek, E. S.; Jusufi, A.; Löwen, H.; Maitland, G. C. J. Phys. Condens. Matter 2002, 14, 9413-9430.

(26) Somerharju, P. J.; Virtanen, J. A.; Eklund, K. K.; Vainio, P.; Kinnunen, P. K. J. Biochemistry 1985, 24, 2773-2781.

(27) Nielsen, S. O. Unpublished data

(28) Press, W. H.; Teukolsky, S. A.; Vetterling, W. T.; Flannery, B. P. Numerical Recipes in Fortran 77: The Art of Scientific Computing, 2nd ed.; Cambridge University Press: New York, 1992. 